\title{
Heartbeat: Prediction of coronary disease risk with cardiac troponin in the general population
}

Cardiac troponin measurements are routinely used for diagnosis and prediction of prognosis in patients presenting with an acute coronary syndrome. ${ }^{1-3}$ Newer highsensitivity assay methods now allow detection of very low levels of serum cardiac troponin (hs-cTnl) but the clinical significance of these low troponin values in asymptomatic patients is unknown. In a cohort of 1135 asymptomatic adults without known atherosclerotic disease, Iribarren and colleagues examined the association between baseline hs-cTnl levels and coronary heart disease (CHD) events over a mean follow-up of 11.3 years (see page 1177). Low levels of troponin were detectable in all subjects (figure 1) and a baseline hs-cTnl $>5.5 \mathrm{ng} /$ $\mathrm{L}$ in men, $>4.2 \mathrm{ng} / \mathrm{L}$ in women was associated with increased risk of subsequent CHD compared with the bottom quartile (HR 2.47, 95\% CI 1.55 to 3.93), even after adjustment for age, sex, race, ethnicity, education level, diabetes, C-reactive protein, renal function and Framingham risk score. Overall, CHD risk category was re-classified in $18 \%$ of patients when hs-cTnl was added to the Framingham risk score.

In the accompanying editorial, Shah (see page 1153) suggests "It is biologically implausible that circulating troponins cause increased risk of coronary disease; instead they may reflect the severity of the underlying atherosclerosis or subclinical myocardial ischaemia, or may be proxies for conditions associated with increased cardiovascular risk such as renal impairment." Given the low event rate in this and previous studies, Shah further recommends: "Larger population-based cohort studies, with comprehensive risk factor and biomarker measurement, would be required to fully assess the clinical utility of troponins in cardiovascular risk prediction".

Cardiac rehabilitation (CR) is an effective approach for improving long term outcome after an acute coronary event. ${ }^{4}$ However, participation rates are low, typically only $10-30 \%$, due to several

Correspondence to Professor Catherine M Otto, Division of Cardiology, University of Washington, Seattle,WA 98195, USA; cmotto@u.washington.edu

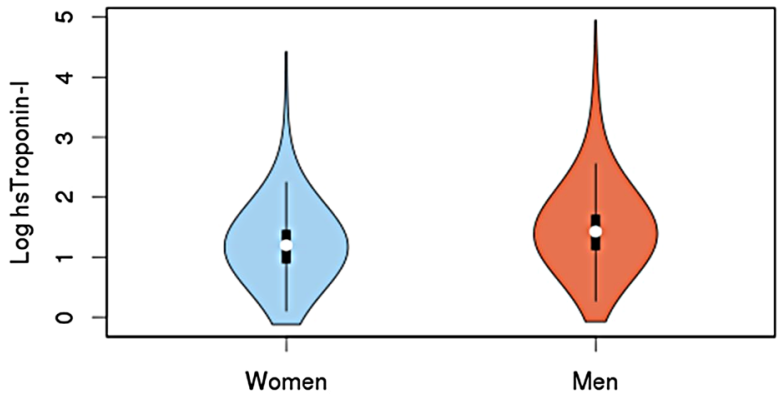

Figure 1 Violin plots showing the distribution of high-sensitivity cardiac troponin I (hs-cTnl), in loge-scale, in men and women. Violin plots show the relative data distribution by the curved (violin-shaped) lines with a standard box-plot embedded within the violin plot.

barriers, including program availability, need for transport, time commitment, and cost. $^{5}$ Telehealth uses current information and communication technologies to provide exercise cardiac rehabilitation (exCR) without the need for scheduled appointments at a supervised exercise facility. ${ }^{6-8}$ In a rigorous systematic review and meta-analysis of 11 randomized controlled clinical trials (1189 patients) telehealth exCR, compared to centre-based exCR or usual care, resulted in higher physical activity levels, better exercise adherence, a lower diastolic blood pressure and lower low-density lipoprotein cholesterol level, with comparable effectiveness for maximal aerobic exercise capacity and other modifiable cardiovascular risk factors (see page 1183) (figure 2).

Dalal and Taylor (see page 1155) comment "Use of telehealth-based CR models will help to augment current centre-based and home-based CR interventions to reach a much larger population with coronary heart disease. It is important to remember that patients can have strong preferences for the model of
CR they receive. Where possible these choices of centre, home and telehealth (and combinations) need to be considered by providers and commissioners to address our current uptake challenge in CR."

In order to better understand the effect of HIV-infection on cardiovascular disease (CVD) risk, a cross sectional study in Tanzania calculated cardiovascular risk scores in HIV-infected patients receiving and not receiving antiretroviral therapy (ART) compared to controls (see page 1200). Although 10-year risk scores were similar, lifetime CVD risk was higher in HIV-infected patients on ART compared to controls $(34.7 \%$ vs $17.0 \%, \mathrm{p}<0.001)$. In addition, prevalence of metabolic syndrome was high and almost 29\% of patients met criteria for statin therapy.

In an editorial Feinstein points out (see page 1157) the limitations of the use of predicted CVD risk as the proxy outcome measure in this study - "predicted risk is not actual risk." Even so, "The primary contributions of this study to literature on cardiovascular complications of HIV are twofold: (1) the findings are congruent with those

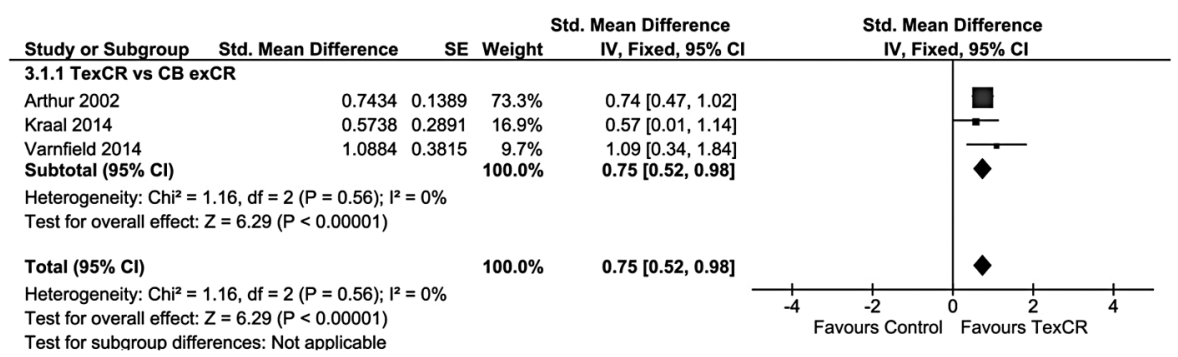

Figure 2 Forest plot for exercise adherence. CBexCR, centre-based exercise-based cardiac rehabilitation; TexCR, telehealth exercise-based cardiac rehabilitation. 


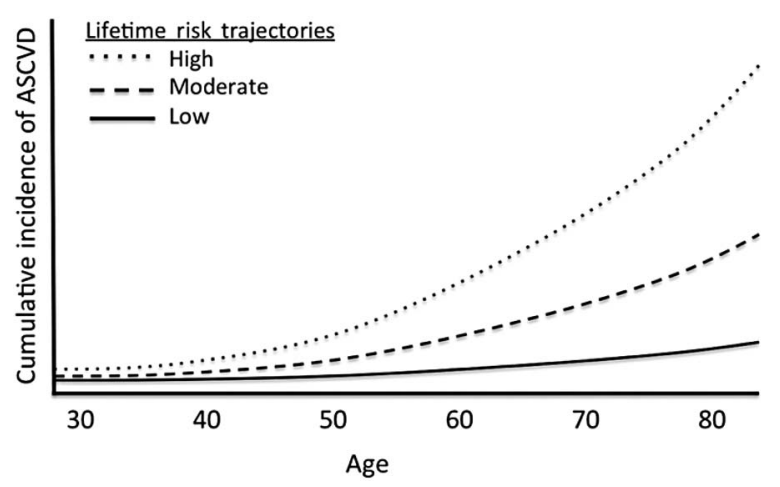

Figure 3 Conceptual model of lifetime atherosclerotic cardiovascular disease (ASCVD) risk trajectories. ASCVD risks increase with age. Because absolute ASCVD risks are relatively low in young adulthood but increase with age, short-term ASCVD risks for high-risk versus low-risk strata may not appear different during young adulthood despite dramatically different cumulative ASCVD risks over the life course.

from previous studies finding a relatively high prevalence of metabolic syndrome among HIV-infected persons on ART and (2) the authors are to be commended on thinking beyond the 10-year risk assessment paradigm in HIV" (figure 3).

The Education in Heart article in this issue (see page 1230) provides a concise review of cardiac masses and tumours. When a cardiac mass is identified, we should remember that the likelihood of a secondary tumor is 20 times higher than the likelihood of a primary cardiac tumor. Most primary cardiac tumors are benign with myxoma being the most common. Although the presenting signs and symp- toms of a cardiac tumor are varied and nonspecific, multimodality imaging allows prompt and accurate diagnosis as so nicely illustrated, with pathologic correlation, in this article. The Image Challenge case in this issue shows a rare diagnosis but the images are intriguing (see page 1199).

Cardiology in Focus is a new feature in Heart that will present short pieces aimed towards providing career advice and mentorship for researchers, trainees and clinical cardiologists, as well as the occasional topic of general interest. Our first article is an interview with Professor Peter Weissberg, Medical Director of the British Heart Foundation (see page 1247).

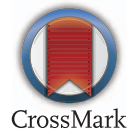

To cite Otto CM. Heart 2016;102:1151-1152.

Heart 2016;102:1151-1152.

doi:10.1136/heartjnl-2016-310190

\section{REFERENCES}

1 Body R, Carley S, McDowell G, et al. The Manchester Acute Coronary Syndromes (MACS) decision rule for suspected cardiac chest pain: derivation and external validation. Heart 2014;100:1462-8.

2 Carlton EW, Cullen L, Than M, et al. A novel diagnostic protocol to identify patients suitable for discharge after a single high-sensitivity troponin. Heart 2015;101:1041-6.

3 Sanchis J, García-Blas S, Mainar L, et al. High-sensitivity versus conventional troponin for management and prognosis assessment of patients with acute chest pain. Heart 2014;100:1591-6.

4 Taylor RS, Anderson LJ. Cochrane corner: cardiac rehabilitation for people with heart disease. Heart 2015; 101:1256-60

5 Redfern J, Hyun K, Chew DP, et al. Prescription of secondary prevention medications, lifestyle advice, and referral to rehabilitation among acute coronary syndrome inpatients: results from a large prospective audit in Australia and New Zealand. Heart 2014;100:1281-8.

6 Varnfield M, Karunanithi M, Lee CK, et al. Smartphone-based home care model improved use of cardiac rehabilitation in postmyocardial infarction patients: results from a randomised controlled trial. Heart 2014;100:1770-9.

7 Turk-Adawi K, Grace SL. Smartphone-based cardiac rehabilitation. Heart 2014;100:1737-8.

8 Clark AM, Redfern J, Briffa T. Cardiac rehabilitation: fit to face the future? Heart 2014;100:355-6. 